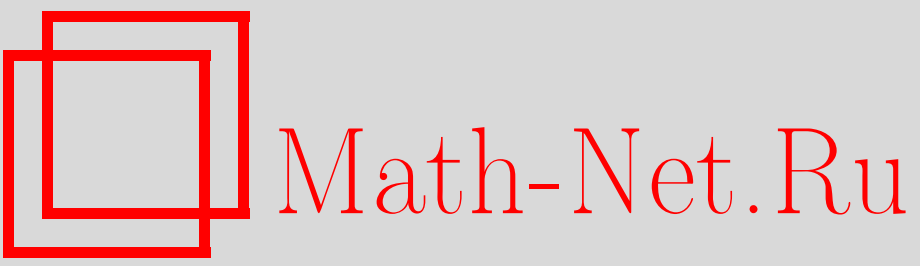

Л. О. Чехов, Наблюдаемые в $2+1$ гравитации и некоммутативные пространства Тейхмюллера, ТМФ, 2001, том 129, номер 2, 360-368

DOI: https://doi.org/10.4213/tmf542

Использование Общероссийского математического портала Math-Net.Ru подразумевает, что вы прочитали и согласны с пользовательским соглашением

http://www.mathnet.ru/rus/agreement

Параметры загрузки:

IP: 54.209 .52 .79

26 апреля 2023 г., $16: 16: 46$ 
ТЕОРЕТИЧЕСКАЯ

И МАТЕМАТИЧЕСКАЯ

ФИЗИКА

Том 129, № 2

ноябрь, 2001

(C) 2001 г.

Л.О. Чехов*

\section{НАБЛЮДАЕМЫЕ В $2+1$ ГРАВИТАЦИИ И НЕКОММУТАТИВНЫЕ ПРОСТРАНСТВА ТЕЙХМЮЛЛЕРА}

Алгебра квантовых геодезических, полученная квантованием координат пространства Тейхмюллера, оказывается квантовой алгеброй $s o_{q}(m)$ Нельсон и Редже. Эта алгебра является алгеброй монодромий (вильсоновских петель) в теории Черна-Саймонса и дает эффективное описание $(2+1)$-мерной гравитации.

\section{1. ВВЕДЕНИЕ}

В настоящей работе описывается алгебра наблюдаемых квантовой 3D гравитации. Фазовое пространство классической эйнштейновой гравитации в $3 \mathrm{D}$ многообразии есть пространство Тейхмюллера границы этого многообразия [1]. На этом пространстве может быть задана каноническая пуассонова структура (структура Вейля-Петерсона), группа симметрий которой есть группа классов отображений. При этом алгебра наблюдаемых соответствуюшей квантовой теории есть некоммутативная деформация *-алгебры функций, задаваемая деформацией пуассоновой структуры, а группа симметрий задает автоморфизмы алгебры наблюдаемых. Такое квантование - построение семейства *-алгебр, зависяших от параметра квантования $\hbar$, и действия группы классов отображений на этом семействе - было проделано в работах [2], [3]. Наблюдаемые в этой картине суть длины замкнутых геодезических на римановой поверхности, и для того чтобы выразить эти наблюдаемые через координаты пространства Тейхмюллера, использовалась техника графов.

В данной работе показывается, что пространства Тейхмюллера римановых поверхностей с дырками естественным образом возникают в трехмерной геометрии и для поверхностей произвольного рода с одной и двумя дырками полученная алгебра квантовых геодезических поднимается с пуассонова листа квантовой алгебры $s o_{q}(m)$ [4].

\section{2. ФОРМАЛИЗМ ПЕРВОГО ПОРЯДКА В 3D ГРАВИТАЦИИ}

Опишем подход к $(2+1)$-мерной гравитации Нельсон, Редже и Цертуша [4], [5], основанный на формализме первого порядка. Классические решения стандартного действия

* Математический институт им. В. А. Стеклова РАН, Москва, Россия. E-mail: chekhov@mi.ras.ru 
Эйнштейна-Гильберта (без материи)

$$
I=\frac{1}{16 \pi G} \int_{M} d^{3} x \sqrt{-g}(R-2 \Lambda)
$$

задаются пространствами постоянной кривизны с топологией $M \sim[0,1] \times S$, где $S$ - это 2D поверхность (в первоначальной формулировке замкнутая).

В формализме первого порядка основные переменные - это спиновая связность $w_{\mu}^{a b}$ и локальная тетрада ("dreibein") $e_{\mu}^{a}$ такая, что $\eta_{a b} e_{\mu}^{a} e_{\nu}^{b}=g_{\mu \nu}, \eta_{a b}=\operatorname{diag}\{-++\}$. Наборы величин $e$ и $w$ считаются независимыми переменными, и как $e$, так и $w$ представляют собой 1-формы $e^{a}=e_{\mu}^{a} d x^{\mu}$ и $w^{a}=(1 / 2) \epsilon^{a b c} w_{\mu b c} d x^{\mu}$. Тогда действие (2.1) принимает вид

$$
I=-2 \int_{M}\left(e \wedge d w+\frac{1}{2} e \wedge w \wedge w+\frac{\Lambda}{6} e \wedge e \wedge e\right),
$$

и при $\Lambda=-1 / \ell^{2}<0$ можно ввести переменные $A^{( \pm) a}=w^{a} \pm e^{a} / \ell$ и получить теорию Черна-Саймонса (ЧС) с калибровочным потенциалом $S O(2,1) \times S O(2,1)$ :

$$
I\left[A^{(+)}, A^{(-)}\right]=I_{\mathrm{CS}}\left[A^{(+)}\right]-I_{\mathrm{CS}}\left[A^{(-)}\right]
$$

где

$$
I_{\mathrm{CS}}[A]=\frac{k}{4 \pi} \int_{M} \operatorname{tr}\left(A \wedge d A+\frac{2}{3} A \wedge A \wedge A\right)
$$

- обычное действие ЧС ( $k$ - константа действия).

Скобки Пуассона двух динамических (пространственноподобных) компонент имеют вид [4]

$$
\left\{A_{i}^{( \pm) a}(x), A_{j}^{( \pm) b}\left(x^{\prime}\right)\right\}= \pm \frac{1}{\ell} \epsilon_{i j} \eta^{a b} \delta^{2}\left(x-x^{\prime}\right),
$$

а переменные $A^{+}$пуассоново коммутируют с переменными $A^{-}$. Голономии, или геодезические функции, имеют вид

$$
G_{\gamma}^{( \pm)}=P \exp \left\{\int_{\gamma} A_{i}^{( \pm) a} T_{a} d x^{i}\right\},
$$

и нетрудно найти их скобки Пуассона. Ввиду топологичности теории кривая $\gamma$ может быть выбрана геодезической кривой. Эти скобки так же, как и распутывающие соотношения, будут получены ниже в альтернативном подходе с использованием техники графов.

\section{3. КЛАССИЧЕСКИЕ ПРОСТРАНСТВА ТЕЙХМЮЛЛЕРА. ТЕХНИКА ГРАФОВ}

В классическом описании пространства Тейхмюллера $\mathcal{T}_{g, n}$ римановых поверхностей рода $g$ с $n$ дырками $(n>0)$ суть пространства комплексных структур на (возможно открытых) римановых поверхностях $S$ по модулю диффеоморфизмов, гомотопически-эквивалентных тождественному преобразованию. В окрестности граничной компоненты комплексная структура изоморфна, как комплексное многообразие, либо кольцу (дырка), либо проколотому диску (выколотая точка). 
Ориентируемая 2D поверхность может быть непрерывно конформно преобразована к пространству постоянной кривизны. Теорема униформизации по Пуанкаре утверждает, что любая комплексная поверхность $S$ постоянной отрицательной кривизны есть верхняя полуплоскость $\mathbb{H}_{+}$с гиперболической метрикой $d s^{2}=d z d \bar{z} /(\operatorname{Im} z)^{2}$, профакторизованная относительно действия дискретной фуксовой подгруппы $\Delta(S)$ полной группы автоморфизмов $P S L(2, \mathbb{R})$,

$$
S=\mathbb{H}_{+} / \Delta(S) .
$$

Каждый класс гомотопий замкнутых кривых $\gamma$ содержит единственную замкнутую геодезическую длины $l(\gamma)=\ln \left|\lambda_{1} / \lambda_{2}\right|$, где $\lambda_{1}$ и $\lambda_{2}$ - (различные) собственные значения элемента $P S L(2, \mathbb{R})$, который отвечает $\gamma$. Эти длины и являются нужными физическими наблюдаемыми.

Основным элементом конструкции является описание пространства Тейхмюллера $\mathcal{T}_{g, n}^{h}(S)$ в терминах ленточных графоов [6].

Пусть ленточныи граф Г - это граф с заданным циклическим порядком ребер, входящих в каждую вершину. Пространство Тейхмюллера координатизуется, если присвоить вешественные положительные числа $Z_{\alpha} \in \mathbb{R}$ неориентируемым ребрам графа, получив тем самым набор $\left\{Z_{\alpha} \mid \alpha \in E(\Gamma)\right\}$, в котором $E(\Gamma)$ - множество всех (неориентированных) ребер графа Г. Мы также обозначим через $V(\Gamma)$ множество вершин графа $\Gamma$.

Ленточный граф, вложенный в ориентированную поверхность, наследует каноническую ленточную структуру согласно ориентации поверхности. Обозначим через $\Gamma(S)$ множество всех изотопических классов трехвалентных ленточных графов, отображенных на поверхность $S$ таким образом, что поверхность становится стягиваемой к граничным компонентам. Каждому графу $u \in \Gamma(S)$ и каждому ребру граффа $u$ можно сопоставить стандартный морфизм $u \mapsto v$, где $v \in \Gamma(S)$ получается из $u$ с помошью флипа данного ребра (см. ниже рис. 1). Множество таких морфизмов порождает группу классов отображсний.

Таким образом, трехвалентный граф Г рода $g$ с $n$ дырками порождает изоморфизм меж ду множеством точек пространства $\mathcal{T}_{g, n}(S)$ и множеством $\mathbb{R}^{\# \text { edges }}$ ребер этого графа, снабженных вешественными числами [6].

Для того чтобы параметризовать замкнутые геодезические (nymu вдоль ребер графа) на римановой поверхности, ассоциируем матрицу $X_{Z_{\alpha}} \in P S L(2, \mathbb{R})$ преобразования Мёбиуса с каждым ребром $\alpha$ :

$$
X_{Z_{\alpha}}=\left(\begin{array}{cc}
0 & -e^{\frac{Z_{\alpha}}{2}} \\
e^{-\frac{Z_{\alpha}}{2}} & 0
\end{array}\right)
$$

и введем матрицы “правых" и “левых" поворотов

$$
R=\left(\begin{array}{cc}
1 & 1 \\
-1 & 0
\end{array}\right), \quad L \equiv R^{2}=\left(\begin{array}{cc}
0 & 1 \\
-1 & -1
\end{array}\right) .
$$

Будем называть замкнутым путем в графе Г любой ориентированный путь, который начинается и заканчивается на одном и том же ребре графа. Произведение матриц, ассоциированных с последовательными ребрами и поворотами этого пути, имеет вид

$$
P_{Z_{1} \ldots Z_{n}}=L X_{Z_{n}} L X_{Z_{n-1}} R X_{Z_{n-2}} \ldots R X_{Z_{2}} L X_{Z_{1}} .
$$


ПРЕДЛОЖЕНИЕ 1 [6]. Существует взаимно однозначное соответствие между элементами множества классов сопряженности ориентированных замкнутых путей $P_{Z_{1}} \ldots Z_{n}$ и замкнутыми (ориентированными) геодезическими $\gamma$ на пространстве Тейхмюллера; при этом длина геодезической $L_{\gamma}$ определяется соотношением

$$
G_{\gamma} \equiv 2 \operatorname{ch}\left(\frac{L_{\gamma}}{2}\right)=\operatorname{tr} P_{Z_{1} \ldots Z_{n}}
$$

где $P_{Z_{1} \ldots Z_{n}}$ - единственный замкнутый путь без возвращений назад, который отвечает данному классу сопряженности ориентируемых путей в Г. Величинь $G_{\gamma}$ называются геодезическими функииями.

В координатах $Z_{\alpha}$ каноническая пуассонова структура $B_{\mathrm{WP}}$ (структура Петерсона-Вейля) на $\mathcal{T}_{g, n}^{h}(S)$ имеет вид

$$
B_{\mathrm{WP}}=\sum_{v \in V(\Gamma)} \sum_{i=1}^{3} \frac{\partial}{\partial Z_{v_{i}}} \wedge \frac{\partial}{\partial Z_{v_{i+1}}}
$$

где $v_{i}, i=1,2,3(\bmod 3),-$ номера циклически ориентированных ребер, входящих в вершину $v$. Эта структура вырожденна, и ее функции Казимира суть длины геодезических вокруг дырок.

Преобразование, сохраняющее пуассонову структуру (3.4) при операции флипа и удовлетворяющее тождеству пятиугольника, изображено на рис. 1 , где

$$
M_{Z}:\{A, B, C, D, Z\} \rightarrow\{A+\phi(Z), B-\phi(-Z), C+\phi(Z), D-\phi(-Z),-Z\}
$$

и в классическом случае $[6] \phi(Z)=\ln \left(e^{Z}+1\right)$.

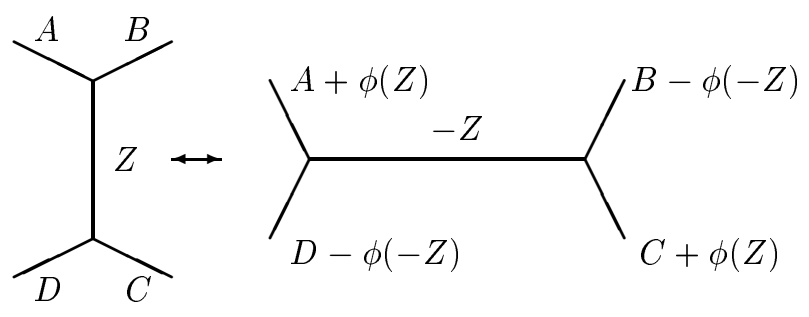

Рис. 1

ЛЕмма. Преобразование (3.5) оставляет неизменными следы произведений по путям (3.3), а потому классические длины геодезических инвариантны относительно действия группь классов отображений.

Геодезические функции $G_{\gamma}(3.3)$, исследованные в работе [7], образуют алгебру (по отношению к произведению и к скобке Пуассона) над $\mathbb{Z}$.

Рассмотрим теперь пуассонову структуру геодезических функций. Для двух непересекающихся геодезических пуассонова скобка равна нулю. Поскольку пуассонова скобка $\left\{G_{1}, G_{2}\right\}$ двух геодезических функций удовлетворяет правилу Лейбница, достаточно 
рассмотреть только лишь простое пересечение геодезических. В графическом виде тогда получим соотношение

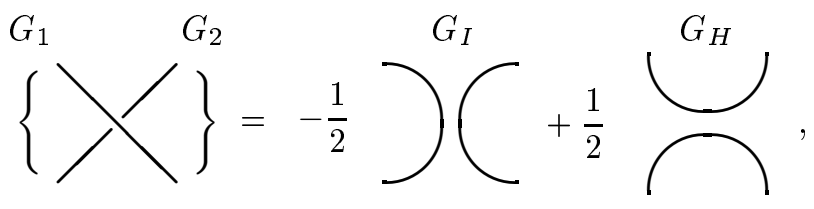

полученное в работе [7] в непрерывной параметризации.

Классическое распутьваюшее соотношение следует из формулы $\operatorname{tr}(A B)+\operatorname{tr}\left(A B^{-1}\right)-$ $\operatorname{tr} A \cdot \operatorname{tr} B=0$, которая верна для произвольных $(2 \times 2)$-матриц $A$ и $B$ с единичными детерминантами. Введем дополнительный множитель \# $G$ для подграфа. Этот множитель равен числу геодезических в полном графе, получаемом как объединение данного подграффа и оставшейся части граффа, которая предполагается одинаковой для всех графов, входящих в соотношение. Имеем

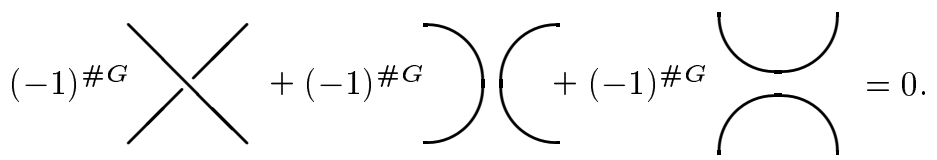

Назовем геодезическую, проходящую по каждому ребру графа не более одного раза, простой геодезической. Набор простых геодезических тем самым конечен и зависит от комбинаторного типа графа.

Пример 1. Для тора $\left(\mathcal{T}_{1,1}\right)$ имеются три переменные $X, Y, Z$ такие, что

$$
\{X, Y\}=\{Y, Z\}=\{Z, X\}=2
$$

(элемент Казимира равен $X+Y+Z$ ). Геодезические функции для трех простых геодезических имеют вид

$$
\begin{aligned}
& G_{X}=\operatorname{tr} L X_{Y} R X_{Z}=e^{-\frac{Y}{2}-\frac{Z}{2}}+e^{-\frac{Y}{2}+\frac{Z}{2}}+e^{\frac{Y}{2}+\frac{Z}{2}} \\
& G_{Y}=\operatorname{tr} L X_{Z} R X_{X}=e^{-\frac{Z}{2}-\frac{X}{2}}+e^{-\frac{Z}{2}+\frac{X}{2}}+e^{\frac{Z}{2}+\frac{X}{2}} \\
& G_{Z}=\operatorname{tr} L X_{X} R X_{Y}=e^{-\frac{X}{2}-\frac{Y}{2}}+e^{-\frac{X}{2}+\frac{Y}{2}}+e^{\frac{X}{2}+\frac{Y}{2}} .
\end{aligned}
$$

Определяя геодезическую функцию $\widetilde{G}_{Z}$, получаемую из $G_{Z}$ преобразованием флипа,

$$
\widetilde{G}_{Z}=\operatorname{tr} R X_{Z} R X_{X} L X_{Z} L X_{Y}=e^{-\frac{X}{2}-\frac{Y}{2}-Z}+e^{\frac{X}{2}-\frac{Y}{2}}\left(e^{-Z}+e^{Z}+2\right)+e^{\frac{X}{2}+\frac{Y}{2}+Z},
$$

получим, что $\left\{G_{X}, G_{Y}\right\}=\widetilde{G}_{Z} / 2-G_{Z} / 2$, и поскольку из соотношений (3.7) следует, что $G_{X} G_{Y}=G_{Z}+\widetilde{G}_{Z}$, получим формулу

$$
\left\{G_{X}, G_{Y}\right\}=\frac{1}{2} G_{X} G_{Y}+G_{Z},
$$

т.е. классическая пуассонова алгебра оказывается замкнутой на наборе $\left\{G_{X}, G_{Y}, G_{Z}\right\}$ геодезических функций (два других соотношения получаются из (3.9) циклическими перестановками). Цена этого замыкания - появление квадратичных элементов в правой части соотношения. 


\section{4. КВАНТОВАНИЕ}

Обозначим через $\mathcal{T}_{g, n}^{\hbar}(\Gamma)$, где $\Gamma \in \Gamma(S)$, алгебру, порождаемую генераторами $Z_{\alpha}^{\hbar}$ (по одному генератору на каждое (неориентированное) ребро $\alpha$ ), с соотношениями

$$
\left[Z_{\alpha}^{\hbar}, Z_{\beta}^{\hbar}\right]=2 \pi i \hbar\left\{Z_{\alpha}, Z_{\beta}\right\}
$$

(cр. (3.4)) и с $*$-структурой $\left(Z_{\alpha}^{\hbar}\right)^{*}=Z_{\alpha}^{\hbar}$. В этих соотношениях $Z_{\alpha}$ и $\{\cdot, \cdot\}$ суть соответствуюшие координатные функции и скобка Пуассона на классическом пространстве Тейхмюллера.

Свяжем теперь с каждым преобразованием (морфизмом) флипа гомоморфизм соответствующих *-алгебр, удовлетворяющий соотношению $M_{Z}^{2}=I$ (рис. 1 ) и пятичленному соотношению. Такой морфизм задается отображением (3.5), в которое надо подставить (квантовую) функцию флипа [8], [9]

$$
\phi(z) \equiv \phi^{\hbar}(z)=-\frac{\pi \hbar}{2} \int_{\Omega} \frac{e^{-i p z}}{\operatorname{sh}(\pi p) \operatorname{sh}(\pi \hbar p)} d p
$$

где контур $\Omega$ проходит по вешественной оси и обходит начало координат сверху.

Для каждого контура $\gamma$ определим оператор квантовой геодезической $G_{\gamma} \in \mathcal{T}^{\hbar}$ соотношением

$$
G_{\gamma} \equiv \underset{\times}{\times} \operatorname{tr} P_{Z_{1} \ldots Z_{n}} \stackrel{\times}{\times} \equiv \sum_{\substack{j \in J \\ \kappa \in\{j\}}} \exp \left\{\frac{1}{2} \sum_{\alpha \in E(\Gamma)}\left(m_{j}(\gamma, \alpha) Z_{\alpha}^{\hbar}+2 \pi i \hbar c_{j}^{\kappa}(\gamma, \alpha)\right)\right\},
$$

в котором квантовое упорядочение $\underset{\times}{\times} \cdot \underset{\times}{\times}$ означает, что классические формулы подправляются с помошью введения дополнительных целых коэффишиентов $c_{j}^{\kappa}(\gamma, \alpha)$, которые определяются из следуюших условий.

1. Набор квантовых геодезических $\left\{G_{\gamma}\right\}$ должен быть инвариантен относительно действия группы классов отображений $\Delta(S)(3.5)$, т.е. для любого морфизма $\delta \in$ $\Delta(S)$ и замкнутого пути $\gamma$ должно выполняться условие $\delta\left(G_{\gamma}\right)=G_{\delta \gamma}$.

2. Алгебра геодезических. Произведение двух квантовых геодезических выражается в виде линейной комбинации квантовых геодезических ламинаций (КГЛ), подчиняюшихся квантовому распутываюшему соотношению [10]. Аналогично классическому случаю, КГЛ есть набор не(само)пересекаюшихся квантовых геодезических.

3. Неориентированность. Квантовые следы операторов прямой и обратной геодезических совпадают.

4. Степени геодезических. Квантовая геодезическая $G_{n \gamma}$ с неотрицательным числом намоток $n$ выражается через $G_{\gamma}$ в точности так же, как и в классическом случае:

$$
G_{n \gamma}=2 T_{n}\left(G_{\gamma} / 2\right),
$$

где $T_{n}(x)$ - многочлены Чебышева. 
5. Если замкнутые пути $\gamma$ и $\gamma^{\prime}$ не пересекаются, то операторы $G_{\gamma}$ и $G_{\gamma^{\prime}}$ коммутируют. Поэтому порядок вхождения квантовых геодезических в КГЛ неважен.

Обозначим упорядочение Вейля обычным знаком нормального упорядочения : · :, т.е.

$$
: e^{a_{1}} e^{a_{2}} \cdots e^{a_{n}}: \equiv e^{a_{1}+a_{2}+\cdots+a_{n}}
$$

для любого набора $\left\{a_{i}: a_{i} \neq-a_{j}\right\}$.

ПРЕДЛОЖЕНИЕ 2. Для простой геодезической квантовое упорядочение совпадает с вейлевским.

Для двух простых геодезических $G_{1}$ и $G_{2}$ с одним нетривиальным пересечением имеem

$$
G_{1} G_{2}=e^{-\frac{i \pi \hbar}{2}} G_{Z}+e^{\frac{i \pi \hbar}{2}} \widetilde{G}_{Z},
$$

или графически получим квантовое распутываюшее соотношение Тураева [10]:

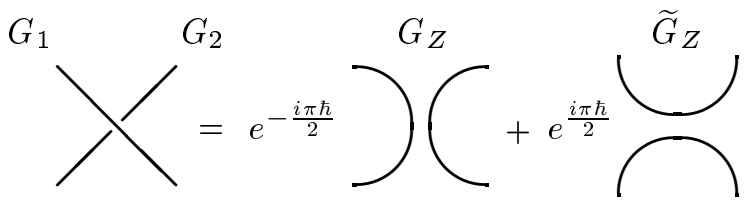

(здесь порядок пересечения $G_{1}$ и $G_{2}$ зависит от того, какая квантовая геодезическая стоит первой в произведении; остаток графа остается одним и тем же для всех величин в формуле (4.6)).

В случае, если число пересечений больше 1 , квантовые распутываюшие соотношения должны применяться одновременно во всех точках пересечения. Это, в частности, приводит к справедливости ридемейстеровских движений в произвольном графе, если положить пустую петлю равной $-e^{-i \pi \hbar}-e^{i \pi \hbar}$.

ПРИМЕР 2. КВАНТОВЫЕ ГЕОДЕЗИЧЕСКИЕ НА ТОРЕ. ДЛя тора ИМеются три Простые квантовые геодезические, задаваемые формулами (3.8) в вейлевски-упорядоченной форме. Квантовая геодезическая $\widetilde{G}_{Z}$, получаемая из $G_{Z}$ преобразованием фолипа,

$$
\widetilde{G}_{Z}=e^{-\frac{X}{2}-\frac{Y}{2}-Z}+e^{\frac{X}{2}-\frac{Y}{2}-Z}+e^{\frac{X}{2}-\frac{Y}{2}} 2 \cos (\pi \hbar)+e^{\frac{X}{2}-\frac{Y}{2}+Z}+e^{\frac{X}{2}+\frac{Y}{2}+Z},
$$

содержит квантовые поправки. Произведение двух простых геодезических имеет вид

$$
G_{X} G_{Y}=e^{\frac{i \pi \hbar}{2}} \widetilde{G}_{Z}+e^{-\frac{i \pi \hbar}{2}} G_{Z}, \quad G_{Y} G_{X}=e^{-\frac{i \pi \hbar}{2}} \widetilde{G}_{Z}+e^{\frac{i \pi \hbar}{2}} G_{Z} .
$$

Обозначив $q \equiv e^{-i \pi \hbar},[A, B]_{q} \equiv q^{1 / 2} A B-q^{-1 / 2} B A$ и $\xi \equiv q-q^{-1}$, получим из (4.7)

$$
\left[G_{X}, G_{Y}\right]_{q}=\xi G_{Z}, \quad\left[G_{Y}, G_{Z}\right]_{q}=\xi G_{X}, \quad\left[G_{Z}, G_{X}\right]_{q}=\xi G_{Y}
$$

что есть так называемая квантовая алгебра $s o_{q}(3)$, исследуемая в работе [11]. Единственный центральный элемент этой алгебры (квантовое соотношение Маркова) имеет вид

$$
\mathcal{M}=G_{X} G_{Y} G_{Z}-q^{\frac{1}{2}}\left(G_{X}^{2}+q^{-2} G_{Y}^{2}+G_{Z}^{2}\right) .
$$




\section{5. КВАНТОВЫЕ АЛГЕБРЫ $s o_{q}(m)$}

Чтобы обобшить пример 2, надо найти графр, для которого простые геодезические составляли бы удобный алгебраический базис. Таким графом является граф, изображенный на рис. 2 , в котором $m$ ребер соединяют $m$ противоположных точек на двух горизонтальных линейных подграфах; двухточечные вершины на концах линейных подграфов мнимы. Простые геодезические в этой картине - это ровно те замкнутые линии, которые проходят в точности по двум различным “вертикальным” ребрам граффа; их поэтому удобно нумеровать индексами этих вертикальных ребер (порядок неважен), и они обозначаются $a_{i j}, i<j$. Пуассонова алгебра геодезических для $a_{i j}$ имеет вид

$$
\left\{a_{i j}, a_{k l}\right\}= \begin{cases}0, & j<k, \\ 0, & k<i, \quad j<l, \\ a_{i k} a_{j l}-a_{k j} a_{i l}, & i<k<j<l, \\ \frac{1}{2} a_{i j} a_{j l}-a_{i l}, & j=k, \\ a_{i l}-\frac{1}{2} a_{i j} a_{i l}, & i=k, \quad j<l, \\ a_{i k}-\frac{1}{2} a_{i j} a_{k j}, & j=l, \quad i<k .\end{cases}
$$

Граф̆ на рис. 2 имеет род $(m / 2)-1$ и две дырки, если $m$ четно, и род $(m-1) / 2$ и одну дырку, если $m$ нечетно. Такие базисы геодезических для четных $m$ и гладких римановых поверхностей рассматривались в работах [4]. При этом полученные пуассоновы алгебры в точности совпадают с (5.1). В математической литературе эта алгебра также возникала как пуассонова алгебра данных монодромии (матриц Стокса) некоторого дифференциального уравнения [12], а также симплектического группоида верхнетреугольных матриц $A$ [13]. Для $(m \times m)$-матриц имеются $[m / 2]$ центральных элементов этой алгебры, порожденных полиномиальными инвариантами $f_{A}(\lambda) \equiv \operatorname{det}\left(A+\lambda A^{\mathrm{T}}\right)=\sum f_{i}(A) \lambda^{i}$.

Алгебра (5.1) была проквантована с помошью метода деформационного квантования в работах [4], [5]. Накладывая коммутационные соотношения (4.1), получим квантовую алгебру $s o_{q}(m)$ работ [5]: $a_{i j}$ коммутируют с $a_{k l}$ при $i<j<k<l$ или при $i<k<l<j$; любая тройка элементов $a_{i j}, a_{j k}$ и $a_{i k}$ образует подалгебру $s_{q}(3)(4.8) ;$ и, наконец, при $i<k<j<l$ получим $\left[a_{i j}, a_{k l}\right]=\xi\left(a_{i k} a_{j l}-a_{i l} a_{j k}\right)$.

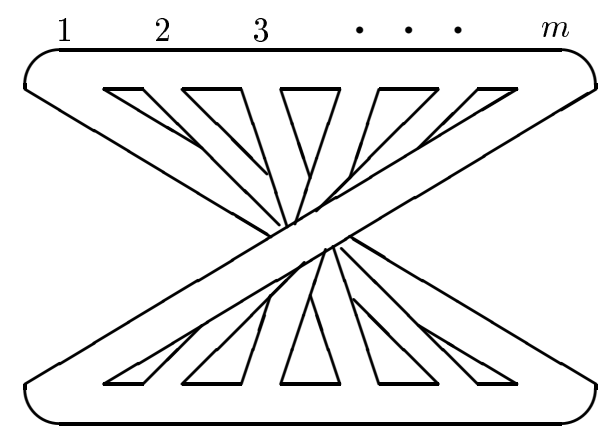

Рис. 2 
Полная пуассонова размерность $d$ алгебры $(5.1)$ равна $m(m-1) / 2-[m / 2]$, и при $m=$ $3,4,5,6, \ldots$ получим $d=2,4,8,12, \ldots$. Размерности соответствуюших пространств Тейхмюллера равны $D=2,4,8,10, \ldots$, и потому пространства Тейхмюллера вкладываются как пуассоновы листы в алгебру (5.1); размерность этих листов перестает быть максимальной только начиная со случая поверхности рода 2 с двумя дырками.

Благодарности. Автор благодарен организаторам конференции за гостеприимство. Работа поддержана грантом РФФИ № 00-02-16477.

\section{Список литературы}

[1] E. Verlinde and H. Verlinde. Conformal field theory and geometric quantization. In: Superstrings 1989. River Edge, NJ: World Scientific, 1990. P. 422.

[2] В. В. Фок, Л. О. Чехов. ТМФ. 1999. Т. 120. № 3. С. 511.

[3] В. В. Фок, Л. О. Чехов. Тр. МИАН. 1999. Т. 226. С. 163.

[4] J. E. Nelson and T. Regge. Nucl. Phys. B. 1989. V. 328. P. 190; J. E. Nelson, T. Regge, and F. Zertuche. Nucl. Phys. B. 1990. V. 339. P. 516.

[5] J. E. Nelson and T. Regge. Phys. Lett. B. 1991. V. 272. P. 213; Commun. Math. Phys. 1993. V. 155. P. 561.

[6] V. V. Fock. Combinatorial description of the moduli space of projective structures. hep-th/9312193.

[7] W. M. Goldman. Invent. Math. 1986. V. 85. P. 263.

[8] L. D. Faddeev. Lett. Math. Phys. 1995. V. 34. P. 249.

[9] R. M. Kashaev. Quantization of Teichmüller spaces and the quantum dilogarithm. q-alg/9705021.

[10] V. G. Turaev. Ann. Scient. Éc. Norm. Sup. Ser. 4. 1991. V. 24. P. 635.

[11] M. Havličck, A.V. Klimyk, and S. Pošta. Representations of the cyclically symmetric $q$-deformed algebra $s_{q}(3)$. math.qa/9805048.

[12] M. Ugaglia. On a Poisson structure on the space of Stokes matrices. math.ag/9902045.

[13] A. Bondal. A symplectic groupoid of triangular bilinear forms and the braid groups. Preprint IHES/M/00/02.

Зав. редакцией Т.Е.Сушко

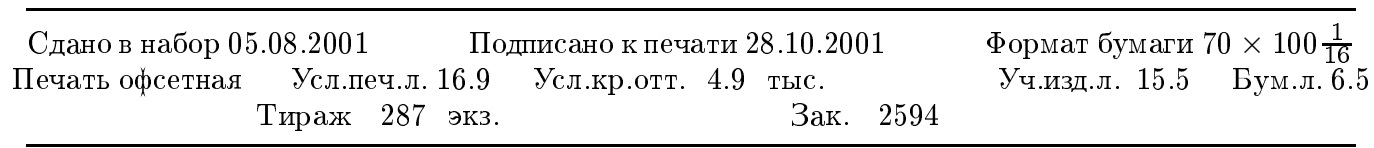

Свидетельство о регистрации № 1876 от 28 мая 1991 года

Зарегистрировано Государственным комитетом СССР по печати

Учредитель: Академия наук СССР, Отделение математики

АДРЕС ИЗДАТЕЛЯ: 117997, Москва, Профсоюзная ул., 90

АДРЕС РЕДАКцИИ: 117966, ГСП, Москва, В-333, ул. Губкина, 8, тел. 135-20-19, e-mail: tmph@mi.ras.ru

Отпечатано в ППП «Типография «Наука», 121099, Москва, Шубинский пер., 6

Налоговая льгота - общероссийский классификатор продукции ОK-005-93, том $2 ; 952000$ - журналы 